\title{
EEG based Emotion Recognition using SVM and LibSVM
}

\author{
Aadhya Bhatt \\ Lakehead University \\ Ontario, Canada
}

\author{
Ananta Bhatt \\ Lakehead University \\ Ontario, Canada
}

\begin{abstract}
In this paper, we solely focus on the EEG dataset. Using SVM classifier with external library LibSVM (3.23), we have classified our EEG SEED dataset and have achieved tremendous improvement in the accuracy and performance. Moreover, we have listed and explained the different approaches followed to improvise the performance and accuracy of our dataset. Further, we have also compared various existing approaches performed on our dataset using various classifiers - ELM, SVM with KNN, SVM with SEED dataset and SVM with DEAP dataset. By using library LibSVM (3.23), we increased the performance of each run by $4 \%$ finally resulting with $79.38 \%$ accuracy having tensor flow environment.
\end{abstract}

\section{Keywords}

SVM, SEED Dataset, KNN, ELM, emotions

\section{INTRODUCTION}

Emotions show a great significance in understanding a person's mental state. It influences both physiological and physical changes which are closely linked to nervous system. In day to day life of humans, emotions play a vital role for social interaction between one another. Further, governs to increase the understanding of the delivered information. Today, many sectors are focusing on emotional recognition for human and machine interaction. Emotions can be largely classified into 6 segments: Happiness, sadness, fear, joy, excitement and anger. Machines should be able recognize and predict these emotions based on mathematical reasoning and logic. In certain cases, such as truth or lie detection, it is difficult to identify real-time emotions with masked sentiments. EEG-based emotion recognition signals record the brain activity in waveform, making it difficult for the subject to fake emotions. These signals focus on 4 frequency patterns: Delta, Theta, Alpha and Beta to diffuse the dominating EEG pattern that characterize the subject in matter. For this research experiment, our focus is on prediction and classification of emotion using the SEED dataset and shallow classifier, support vector machine (SVM). This dataset is a collection of feedback given by 15 participants on 15 Chinese film clips. Video clips are chosen based on certain criteria.

Video should be short, self-explanatory and maximizes the emotion of the participant. Each participant is addressed with 15 seconds hint before the start of the clip and 10 seconds feedback after the end of the clip. These feedbacks are recorded and analysed thoroughly. Here, the emotions are labelled into 3 categories: Positive, Negative and Neutral. The detailed diagram for dataset collection is given below.

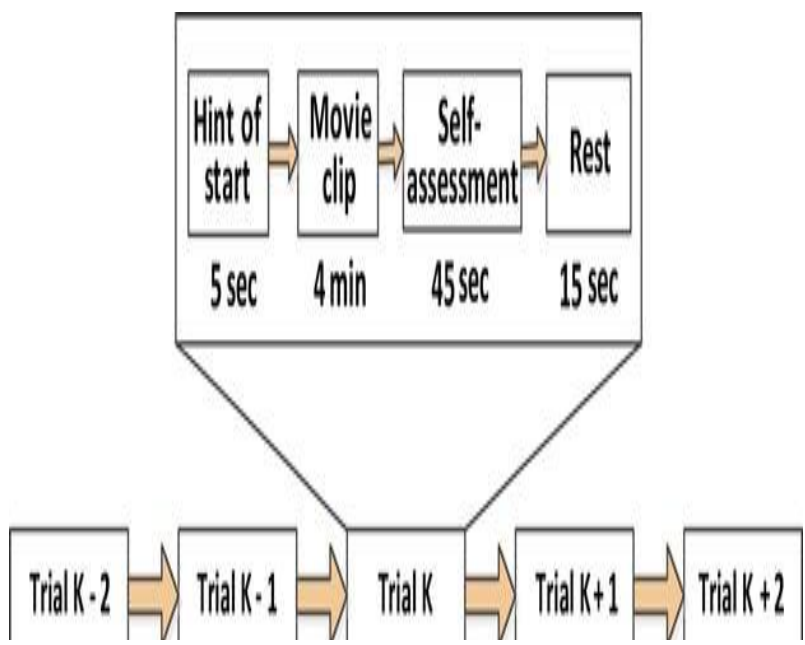

Fig. 1: Detailed Diagram for Dataset collection

This paper incorporates SVM with LibSVM (3.23) library tool extension, an integral software for support vector classification. It provided with direct training methods, parameter selection and scaling of the data. The LibSVM (3.23) method facilitated in achieving tremendous improvement of accuracy when compared to SVM with KNN network.

\section{APPROACHES}

\subsection{Previous Approaches:}

In this section, first we try to compare various existing studies performed with our publicly available EEG dataset. Further, compare the performance of our method with other methods to evaluate the accuracy achieved by the model.

We compare the accuracies obtained with the respective classifiers, which are- ELM, SVM with KNN, SVM with SEED dataset and SVM with DEAP dataset. The calculated mean accuracies achieved by the corresponding classifiers and the dataset are shown in percentage $(\%)$, given in the figure below (Fig. 2).

The average accuracies obtained for ELM network, SVM network with linear kernel and SEED dataset, SVM network with DEAP dataset and SVM with KNN are 86\%, 83.4\%, $82 \%$ and $70.43 \%$ respectively. 


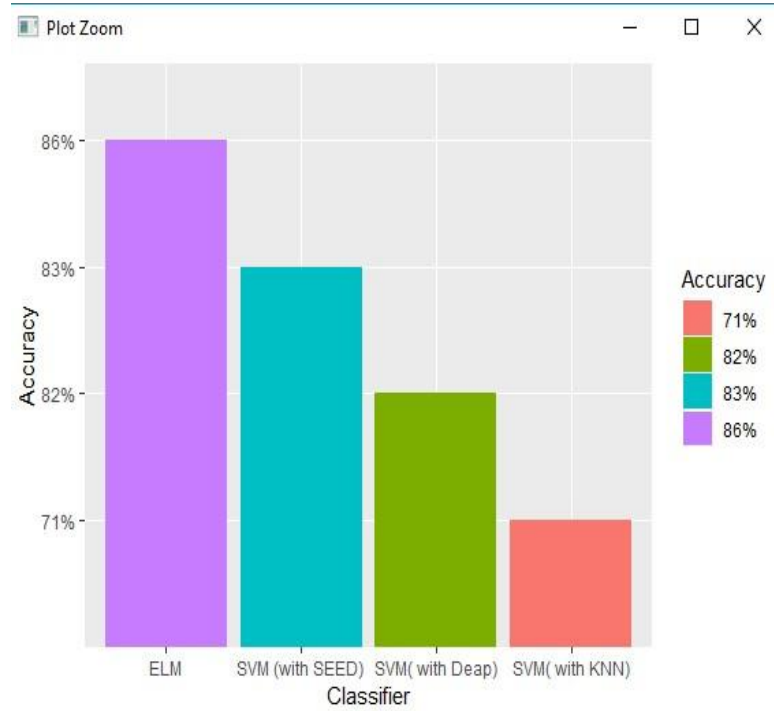

Fig. 2: The average accuracies using different classifiers and dataset

While comparing the above obtained results, we can conclude that ELM marked better performance as compared to other classifiers.

\section{EXPERIMENTS}

In this section, we illustrate the various methods followed in order to improve and achieve better accuracy with our EEG dataset. All the methods performed involved Linear SVM classifier and the accuracy is evaluated from the accuracy rate of the classification. Training dataset through the dataset is about 84420 data whereas, the testing data is 58128 data.

\subsection{First Method (Simple Linear SVM):}

The method involved importing the LinearSVC package file, wherein we tried to fit our training dataset with our training labels. Further, aimed at predicting the test dataset and the test labels. The accuracy scored was calculated using the test data and the predict svm. This method entailed to the following:

batch_size $=128$

num_classes $=10$

epochs $=30$

\subsection{Second Method (Linear SVM with Autoencoder):}

This method is an extension of the above described method, constructed to improvise the efficiency and accuracy of the classification. We first traversed through creating the autoencoder model with 'relu' activation mapping the encoded representation and the 'sigmoid' activation to map the decoded representation. We marked our optimizer to 'adadelta' and loss='binary_crossentropy'. Moreover, fit the training set to the autoencoder using autoencoder.fit():

epochs $=30$ batch_size $=256$

\subsection{Third and Our Method (SVM with LibSVM 3.23):}

This method is governed with an external additional library LibSVM 3.23 which catered to various functions that helped improve the classification tremendously. Certain functions such as: svm_problem() svm_parameter() svm_train() svm_predict()

svm_parameter() which goes along with svm_problem() mapped the training data and the training labels. Further, the svm_parameter() allowed to incorporate objects to set the type of svm, type of kernel and degree in the kernel function and cost with objects as -s, -t, -d, -c respectively. On the contrary, svm_predict() followed the test data and test labels with probability estimates -b.

The below figure, (Fig. 3) gives the detailed flow of the program followed with SVM LibSVM library and the functions entailed with it. With our training data and training label read through with 150 batches.

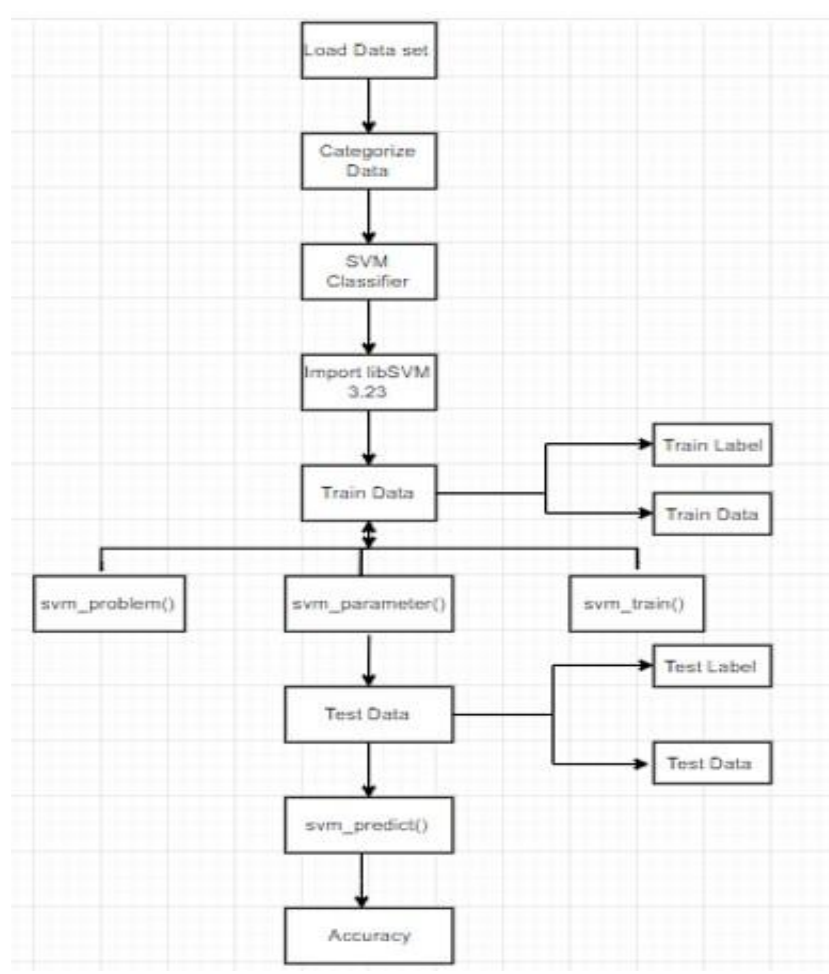

Fig. 3: Basic flow of the program

\subsection{Fourth Method:}

Our last method involved taking parameters from the above method and improvising it by adding autoencoder. In this method, encoded representation involved 'relu' activation set with activity_regularizer and decoded representation required 'sigmoid' activation with channels number set to 310 and the encoding dimension set to 120 . Further, 'sgd' optimizer was set with learning rate as ' 0.01 ' and loss as 'binary_crossentropy'.

The training data is fit using the autoencoder.fit() :

batch_size $=512$ epoch $=100$

\section{RESULT}

As discussed above, all the four methods conducted were aimed at establishing a better performance. However, with the third method the performance drastically improved as shown in table 4 . On the other hand, further improving the method using Autoencoder dramatically decreased the performance as the dataset is comparatively small.

Our third method SVM using LibSVM, with every run the performance increased by almost $4 \%$. After 3 runs the final result given is $79.3852 \%$ as shown in table 5. Further, the 
training time also considerably increased. In this research paper, TensorFlow platform is considered for running our program which contributed to produce increased training time and testing time.

Table. 1: Accuracy of the Classifiers

\begin{tabular}{|c|c|c|}
\hline Sr. no & Classifier & Accuracy \\
\hline 1 & Simple Linear SVM & $39.7861 \%$ \\
\hline 2 & Linear SVM with Autoencoder & $36.321 \%$ \\
\hline 3 & SVM (LibSVM- 3.23) & Training time: $2 \mathrm{hrs} 30$ minutes \\
\hline 4 & SVM (LibSVM-3.23) with Autoencoder & $39 \%$ \\
\hline
\end{tabular}

Table. 2: Accuracy with 3 runs

\begin{tabular}{|c|c|c|c|}
\hline Classifier & Run 1 & Run 2 & Run 3 \\
\hline SVM ( LibSVM- 3.23) & $72.1289 \%$ & $76.685 \%$ & $79.3852 \%$ \\
\end{tabular}

\section{CONCLUSION}

In this paper, we have tried to classify EEG dataset using shallow classifier SVM using LibSVM. We have tried to follow different approaches to enhance the performance and further compared these methods to come up with the best approach.

On the other hand, this research can be used for testing electrical patterns in our brains, for diagnostic conditions such as seizure and epilepsy. It can also be used to confirm brain death. In order to improve the accuracy even further, we can use winners take all approach to classify the dataset into 3 segments-positive, negative and neutral. Another way can be to use other classifiers such as ELM, compatible with our EEG dataset to improvise the accuracy further.

\section{REFERENCES}

[1] Ahmad Tauseef Sohaib, S. Q. (2013). Evaluating Classifiers for Emotion Recognition Using EEG. Springer, 2-8.

[2] Avinash L. Tandle, M. S. (2018). Mental state and emotion detection from musically stimulated EEG. Springer, 3-11.

[3] McOwan, K. A. (2006, February). A Real-Time Automated System for the Recognition. IEEE, 5-7.

[4] Naveen Masood, H. F. (2018). Multimodal Paradigm for Emotion Recognition Based on EEG Signals. Springer, 2-7.

[5] Nisha Vishnupant Kimmatkar, D. V. (2018). Human Emotion Classification from Brain EEG Signal. ACM, $2-4$.

[6] Priyanka S. Ghare, A. P. (2016). Human emotion recognition using non linear and non stationary EEG signal. IEEE, 3-6.

[7] Ruo-Nan Duan, J.-Y. Z.-L. (2013). Differential Entropy Feature for EEGbased Emotion Classification. IEEE 81-84.

[8] S. G. Mangalagowri, P. C. (2016). EEG feature extraction and classification using feed forward backpropogation algorithm for emotion detection. IEEE, 4-7.

[9] S. Thejaswini, K. M. (2017, December 23). EEG Based Emotion Recognition Using Wavelets and Neural Networks Classifier. Springer, 1-6.

[10] Sujata Bhimrao Wankhade, D. D. (2019). IKKN Predictor: An EEG Signal Based Emotion Recognition for HCI. Springer, 1-3.

[11] Wei-Long Zheng, a. B.-L. (2015). Investigating Critical Frequency Bands and Channels for EEG-based Emotion Recognition with Deep Neural Networks. IEEE , 162175 .

[12] Yisi Liu, O. S. (2010). Real-Time EEG-Based Human Emotion Recognition and Visualization. IEEE, 2-7.

[13] Yongbin Gao, H. J. (2015). Deep learninig of EEG signals for emotion recognition. IEEE, 2-4.

[14] Yongbin Gao, H. J. (2015). Deep learninig of EEG signals for emotion recognition. IEEE, 1-4.

[15] z. Khalili, M. H. (2009). Emotion Recognition System Using Brain and Peripheral Signals:Using Correlation Dimension to Improve the Results of EEG. IEEE, 1-4.

[16] Zeynab Mohammadi, J. F. (2015). Wavelet-based emotion recognition system using EEG signal. Springer, $2-5$. 